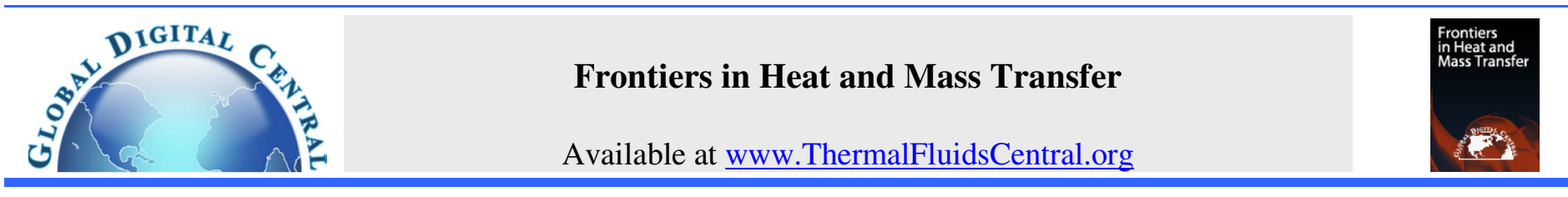

\title{
MIXED CONVECTION FLOW OF NANOFLUID IN A VERTICAL CHANNEL WITH HALL AND ION-SLIP EFFECTS
}

\author{
D. Srinivasacharya*, Md. Shafeeurrahaman \\ Department of Mathematics, National Institute of Technology, Warangal - 506004, India
}

\begin{abstract}
In this article the laminar mixed convective incompressible electrically conducting flow of a nanofluid in vertical channel has been investigated by considering Hall and Ion-slip parameter effects. The nonlinear governing equations are non-dimensionalized and then solving by using HAM procedure. The impact of the magnetic, Hall and Ion-slip parameter on dimensionless velocity, temperature and nanoparticle concentration are investigated and represented geo-metrically.
\end{abstract}

Keywords: $M H D$, Hall and Ion-slip effects, Nanofluid, Vertical channel, HAM

\section{INTRODUCTION}

Heat transfer and mixed convective flow of nanofluid in a vertical channel, a frequently encountered geometry in thermal engineering equipment, has been the concentrating on vast investigation for many years. This is because of its wide range of applications in the structure of cooling devices for electronics and microelectronic equipment, solar energy collection, etc. On the other hand, it is noticed that the convective heat transfer and fluid flow problems with the interaction of magnetite field have increased enormously because of many engineering and industrial applications of these problems. In the investigations concerned with the magnetohydrodynamic (MHD) convective flows, the Hall and Ion-slip terms in Ohm's law were ignored in order to simplify the mathematical analysis of the problem. However, the impact of Hall and Ion-slip are essential in the existence of a strong magnetite field. Therefore, in several physical situations it is required to include the significance of Hall and Ion-slip parameter in the MHD equations. Tani (1962) analyzed the significant effect of Hall current on the electrically conducting viscous fluid flow in vertical channels. Since then several researchers investigated the influence of Hall current and Ion slip effects on the Newtonian and non-Newtonian fluid flow and convective heat transfer in channels. Srinivasacharya and Kaladhar $(2012,2013)$ studied the impact of Hall current and Ion-slip effect on mixed convective couple stress fluid flow through a vertical channel. Manglesh and Gorla (2013) studied the significance of thermal diffusion, thermal radiation and the Halleffect on the unsteady convection flow of an electrically conducting fluid in a vertical channel. Garg et al (2014) analyzed the impact of Hall current on viscoelastic MHD oscillatory convection flow of a fluid in a vertical porous channel.

Convection and heat transfer using nanofluids has acquired considerable attention in present days. It is due to the diverse application in science, engineering and industry such as cooling of nuclear reactor, power generating systems, automobile engines, welding equipment and heat exchanging in electronics devices. Nanofluids, introduced by Choi (1965), are suspensions of nanoparticles in a conventional fluid. Choi (1965) verified that the nanofluids have more thermal-conductivity proportionate to the base fluids. This can be obtained even at very small nanoparticle volume fractions. A number of studies were conducted in recent years, on the flow in a vertical channel filled with nanofluid by considering distinct types of convectional base fluids with particular nanoparticles. Hang and Pop [7] analyzed the fully developed mixed convection flow in a vertical channel filled with nanofluids. Hajipour and Dehkordi (2012) studied the mixed convection and heat transfer flow of a nanofluid in a parallel plates partially filled with highly porous medium. Grosan and Pop (2012) examined the fully developed mixed convection of nanofluid flow in a vertical parallel plates. Sacheti et al. (2012) investigated the transient free convection flow of a nanofluid in a vertical channel. Using the Buongiorno model of nanofluid, Hang et al. (2013) analyzed the mixed convective flow in a vertical channel. Fakour et al. (2014) described the mixed convection flow of a nanofluids in a vertical channel. Sheikholeslami and Anji (2014) considered the MHD flow of a nanofluid in a channel with permeable walls. Makhatar et al. (2015) analyzed the flow of fully developed mixed convection of nanofluid flow in a vertical channel filled with a porous medium. Das et al. (2015) investigated the mixed convective nanofluid flow between vertical parallel plates in an existence of a uniform transverse magnetite field.

The preceding literature reveals that the problem on heat transfer mixed convective flow of nanofluid in a vertical channel considering the impact of Hall and Ion-slip parameters has not been considered. Hence, the aim of the present article is to analyze the significance of Hall and Ion-slip parameter on steady mixed convective heat transfer flow of a nanofluid in a vertical channel. The homotopy analysis method (HAM) is used to solve the nonlinear ordinary differential equations. The HAM, first introduced by Liao (2003), is one of the most powerful technique to solve the various types of strongly nonlinear equations. The effect of flow parameters on the dimensionless velocity, dimensionless temperature and nanoparticle concentration is examined.

\section{MATHEMATICAL FORMULATION}

Consider a steady, laminar, electrically conducting flow of nanofluid passing through a vertical channel. The distance between the plates of the channel is $2 \mathrm{~d}$. The $\mathrm{x}$-axis is appropriated in the direction of the flow vertically upward through the central line of the vertical channel, $y$-axis in the direction orthogonal to the flow as shown in Fig. 1. The plate $y=$ $-d$ is preserved at temperature $T_{l}$ and nanoparticle volume fraction $\phi_{1}$ while plate $y=d$ is preserved at temperature $T_{2}$ and nanoparticle volume fraction $\phi_{2}$. Rate of flow through the plates is $v_{0}$ whichis taken to be

*Corresponding author Email: dsc@ nitw.ac.in, dsrinivasacharya@yahoo.com 
constant, uniform and equal for both the plates. A uniform magnetite field $B_{0}$ is applied in $y$-direction. The Hall current and Ion-slip effects are considered in view of relatively high electron-atom collision frequency. This assumption causes a cross flow in the $z$-direction, therefore the flow becomes three dimensional. The induced magnetite field is neglected in comparison to the applied magnetic field. A uniform pressure gradient in $x$ direction and buoyancy forces caused the mixed convective flow. Further, all the characteristic of fluid are considered as constant apart from the density in the buoyancy term. Assume that the width of the plates along the $\mathrm{x}$ and $\mathrm{z}$ direction is extended infinitely. Hence, the velocity vector of a fluid is taken as $(u(y), v(y), w(y))$. The temperature and nanoparticle volume fraction are respectively denoted by $\mathrm{T}$ and $\phi$.

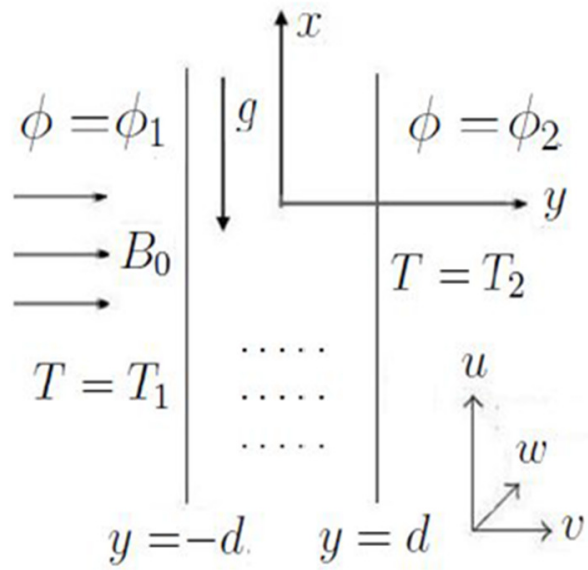

Fig. 1 Schematic representation of the problem

Under these assumptions, the equations governing the nanofluid flow, as proposed by Buongiorno (2006), under the significance of uniform transverse magnetite field are as follows:

$$
\begin{gathered}
\frac{\partial v}{\partial y}=0 \\
\rho_{f} v \frac{\partial u}{\partial y}=-\frac{\partial p}{\partial x}+\mu \frac{\partial^{2} u}{\partial y^{2}}+\left(1-\phi_{0}\right) \rho_{f_{0}} g \beta_{T}\left(T-T_{1}\right) \\
-\left(\rho_{p}-\rho_{f_{0}}\right) g\left(\phi-\phi_{1}\right)-\frac{\sigma B_{0}^{2}}{\left(\alpha_{e}^{2}+\beta_{h}^{2}\right)}\left(\alpha_{e} u+\beta_{h} w\right) \\
\nu \frac{\partial T}{\partial y}=\alpha \frac{\partial^{2} T}{\partial y^{2}}+\frac{2 \mu}{(\rho c)_{p}}\left[\left(\frac{\partial u}{\partial y}\right)^{2}+\left(\frac{\partial w}{\partial y}\right)^{2}\right]+\tau\left[D_{B} \frac{\partial T}{\partial y} \frac{\partial \phi}{\partial y}+\frac{D_{T}}{T_{0}}\left(\frac{\partial T}{\partial y}\right)^{2}\right] \\
v \frac{\partial \phi}{\partial y}=D_{B} \frac{\partial^{2} \phi}{\partial y^{2}}+\frac{D_{T}}{T_{0}} \frac{\partial^{2} T}{\partial y^{2}}
\end{gathered}
$$

Since the rate of flow through the plates is $v_{0}$ and in view of (1), we get $\mathrm{v}=v_{0}(\mathrm{a}$ constant $)$.

The conditions on the boundary are $w=0, u=0, \phi=\phi_{1}, T=T_{1}, \quad$ on $\quad y=-d$, $w=0, u=0, \quad \phi=\phi_{2}, \quad T=T_{2}, \quad$ on $\quad y=d$,

Introducing the following non-dimensional variables

$$
\tilde{w}=\frac{w}{u_{0}}, \tilde{u}=\frac{u}{u_{0}}, \tilde{T}=\frac{T-T_{1}}{T_{2}-T_{1}}, \tilde{\phi}=\frac{\phi-\phi_{1}}{\phi_{2}-\phi_{1}}, P=\frac{d^{2} p}{\mu u_{0}}, \tilde{y}=\frac{y}{d},
$$

in Eqs. (1) - (5), dropping tildes we get

$$
u^{\prime \prime}-R u^{\prime}+\frac{G r}{R e}\left(T-N_{r} \phi\right)-\frac{H a^{2}}{\alpha_{e}{ }^{2}+\beta_{h}{ }^{2}}\left(\alpha_{e} u+\beta_{h} w\right)-A=0
$$

$$
\begin{aligned}
& w^{\prime \prime}-R w^{\prime}+\frac{H a^{2}}{\alpha_{e}^{2}+\beta_{h}^{2}}\left(\beta_{h} u-\alpha_{e} w\right)=0 \\
& T^{\prime \prime}-R P r T^{\prime}+\operatorname{PrN}_{b} T^{\prime} \phi^{\prime}+\operatorname{PrN}_{t} T^{2}+2 B r\left[\left(u^{\prime}\right)^{2}+\left(w^{\prime}\right)^{2}\right]=0 \\
& \phi^{\prime \prime}-R L e \phi^{\prime}+\frac{N t}{N b} T^{\prime \prime}=0
\end{aligned}
$$

where the kinematic viscosity coefficient is $v$, the Prandtl number is $\operatorname{Pr}=\mu C_{P} / k_{f}$, the Lewis number is $L e=v / D_{B}$, the suction/injection parameter is $R=v_{0} d / v$, the Reynold's number is $R e=u_{0} d / v$, Grashof number is $G r=\left((1-\phi) g \beta_{T}\left(T_{2}-T_{1}\right) d^{3}\right) / v^{2}$, the Hartman number is $H a^{2}=B_{0}{ }^{2} d^{2} \sigma / \mu$, the constant pressure gradient is $A=\left(d^{2} / \mu u_{0}\right)(\partial p / \partial x)$, the Brinkman number is $B r=\mu u_{0}^{2} /\left(k_{f}\left(T_{2}-T_{1}\right)\right)$, the Brownian motion parameter is $N b=\tau D_{B}\left(\phi_{2}-\phi_{1}\right) / v$, the thermoporesis parameter is $N t=\tau D_{T}\left(T_{2}-T_{1}\right) / v T_{0}$, buoyancy ratio is $N r=\left(\rho_{p}-\rho_{f_{0}}\right)\left(\phi_{2}-\phi_{1}\right) / \rho_{f_{0}} \beta_{T}\left(T_{2}-T_{1}\right)(1-\phi)$, $\tau=\left(\rho C_{p}\right) /\left(\rho C_{f}\right)$ and $\alpha_{\mathrm{e}}=1+\beta_{h} \beta_{i}$ is a constant.

The corresponding conditions (6) on boundary are

$$
\begin{aligned}
& u=0, \quad w=0, \phi=0, \quad T=0, \quad \text { at } \quad y=-1 \\
& u=0, \quad w=0, \quad \phi=1, \quad T=1, \quad \text { at } \quad y=1
\end{aligned}
$$

\section{NUMERICAL METHOD}

The first step in HAM solution, is choosing the initial approximations of $u(y), w(y), T(y)$ and $\phi(y)$ and auxiliary linear operators.(For more details on the homotopy analysis method see the works of Liao (2003, 2004, 2010, 2013). Therefore, the initial approximations, which are chosen such that they satisfy the boundary conditions, are

$$
u_{0}(y)=0, \quad w_{0}(y)=0, \quad T_{0}(y)=\frac{(y+1)}{2} \quad \text { and } \quad \phi_{0}(y)=\frac{(y+1)}{2}(13)
$$

and the auxiliary linear operators are

$$
L_{i}=\frac{\partial^{2}}{\partial y^{2}} \quad \text { for } \quad i=1,2,3,4
$$

such that

$$
L_{1}\left(c_{1}+c_{2} y\right)=0, L_{2}\left(c_{3}+c_{4} y\right)=0, L_{3}\left(c_{5}+c_{6} y\right)=0 \quad \text { and } \quad L_{4}\left(c_{7}+c_{8} y\right)=0
$$

where $c_{j},(j=1,2, \cdots 8)$ are constants. The second step in HAM is to define the zero ${ }^{\text {th }}$ order deformation, which is given by

$$
\begin{aligned}
& (1-q) L_{1}\left[u(y ; q)-u_{0}(y)\right]=q h_{1} N_{1}[u(y ; q)], \\
& (1-q) L_{2}\left[w(y ; q)-w_{0}(y)\right]=q h_{2} N_{2}[w(y ; q)], \\
& (1-q) L_{3}\left[T(y ; q)-T_{0}(y)\right]=q h_{3} N_{3}[T(y ; q)], \\
& (1-q) L_{4}\left[\phi(y ; q)-\phi_{0}(y)\right]=q h_{4} N_{4}[\phi(y ; q)]
\end{aligned}
$$

where

$$
\begin{aligned}
& N_{1}[\mathrm{u}(y, q)]=u^{\prime \prime}-R u^{\prime}+\frac{G r}{R e}(T-N r \phi)-\frac{H a^{2}}{\alpha_{e}{ }^{2}+\beta_{h}{ }^{2}}\left(\alpha_{e} u+\beta_{h} w\right)-A \\
& N_{2}[w(y, q)]=w^{\prime \prime}-R w^{\prime}+\frac{H a^{2}}{\alpha_{e}{ }^{2}+\beta_{h}{ }^{2}}\left(\beta_{h} u-\alpha_{e} w\right) \\
& N_{3}[T(y, q)]=T^{\prime \prime}-R P r T^{\prime}+\operatorname{PrNb} T^{\prime} \phi^{\prime}+\operatorname{PrNt} T^{\prime 2}+2 \operatorname{Br}\left[\left(u^{\prime}\right)^{2}+\left(w^{\prime}\right)^{2}\right] \\
& N_{4}[\phi(y, q)]=\phi^{\prime \prime}-R L e \phi^{\prime}+\frac{N_{t}}{N_{b}} T^{\prime \prime}
\end{aligned}
$$




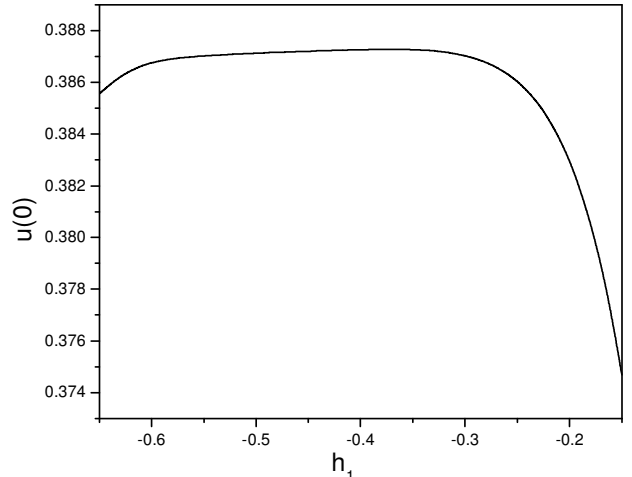

(a) $u(y)$

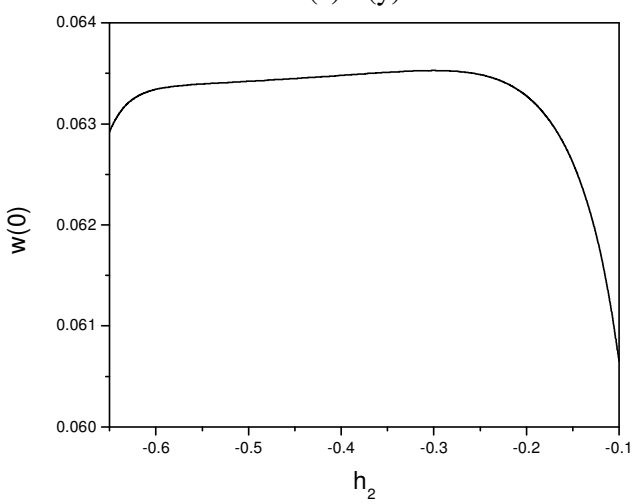

(b) $w(y)$

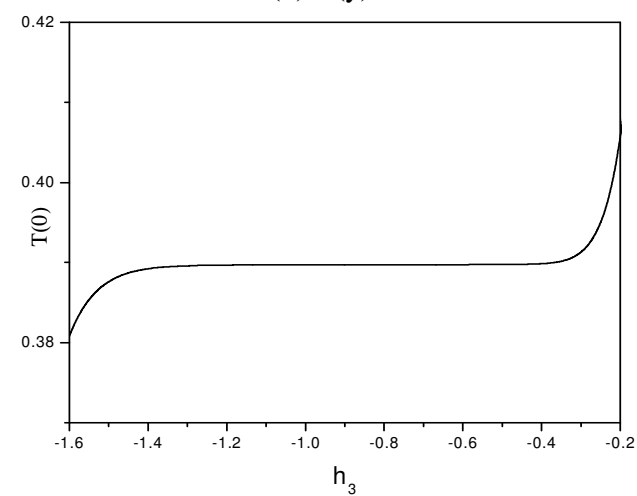

c) $\mathrm{T}(\mathrm{y})$

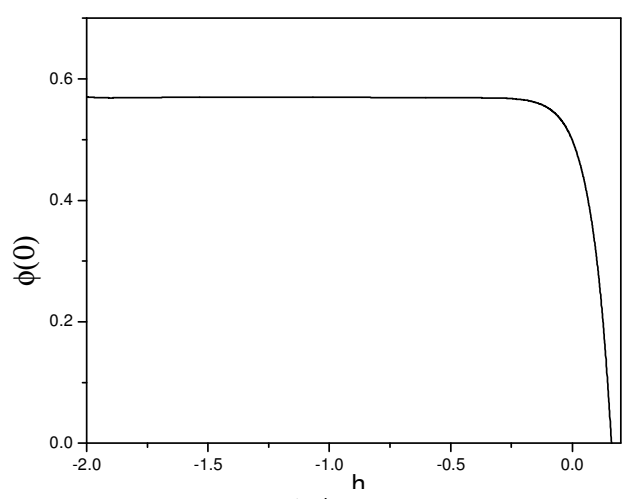

(d) $\phi(y)$

Fig. 2 The $\mathrm{h}$-curve foru(y), w(y), T(y) and $\phi(\mathrm{y})$ for $\mathrm{Le}=1.0, \mathrm{Br}=$ $0.5, \mathrm{Pr}=1.0, \mathrm{Gr}=10, \mathrm{R}=1, \mathrm{Re}=2, \mathrm{Nr}=1.0, \mathrm{Nt}=0.5, \mathrm{Nb}=0.5, \mathrm{Sr}$ $=0.5, \mathrm{Ha}=4.0, \beta_{\mathrm{i}}=2.0, \beta_{\mathrm{h}}=2.0$ and $\mathrm{A}=1$. where $q \in[0,1]$ is the embedded parameter and $h_{i},(i=1,2,3,4$. $)$ are nonzero auxiliary parameters.

The equivalent boundary conditions are

$$
\begin{gathered}
u(-1 ; q)=0, \quad w(-1 ; q)=0, \quad T(-1 ; q)=0, \quad \phi(-1 ; q)=0 \\
u(1 ; q)=0, \quad w(1 ; q)=0, \quad T(1 ; q)=1, \quad \phi(1 ; q)=1
\end{gathered}
$$

Next, the deformation equations of $\mathrm{m}^{\text {th }}$-order are given by

$$
\begin{array}{ll}
L_{1}\left[u_{m}(y)-\chi_{m} u_{m-1}(y)\right]=h_{1} R_{m}^{u}(y), & L_{2}\left[w_{m}(y)-\chi_{m} w_{m-1}(y)\right]=h_{2} R_{m}^{w}(y), \\
L_{3}\left[T_{m}(y)-\chi_{m} T_{m-1}(y)\right]=h_{3} R_{m}^{T}(y), & L_{4}\left[\phi_{m}(y)-\chi_{m} \phi_{m-1}(y)\right]=h_{4} R_{m}^{\phi}(y)
\end{array}
$$

where

$$
\begin{aligned}
& R_{m}^{u}(y)=u^{\prime \prime}-R u^{\prime}+\frac{G r}{R e}(T-N r \phi)-\frac{H a^{2}}{\alpha_{e}{ }^{2}+\beta_{h}{ }^{2}}\left(\alpha_{e} u+\beta_{h} w\right) \\
& -\left(1-\chi_{m}\right) A \\
& R_{m}^{w}(y)=w^{\prime \prime}-R w^{\prime}+\frac{H a^{2}}{\alpha_{e}^{2}+\beta_{h}^{2}}\left(\beta_{h} u-\alpha_{e} w\right) \\
& R_{m}^{T}(y)=T^{\prime \prime}-R \operatorname{Pr} T^{\prime}+\operatorname{PrNb}\left[\sum_{n=0}^{m-1} T_{m-1-n}^{\prime} \phi_{n}^{\prime}\right]+\operatorname{PrNt}\left[\sum_{n=0}^{m-1} T_{m-1-n}^{\prime} T_{n}^{\prime}\right]^{(20)} \\
& +2 B r\left[\sum_{n=0}^{m-1} u_{m-1-n}^{\prime} u_{n}^{\prime}+\sum_{n=0}^{m-1} w_{m-1-n}^{\prime} w_{n}^{\prime}\right] \\
& R_{m}^{\phi}(y)=\phi^{\prime \prime}-R L e \phi^{\prime}+\frac{N_{t}}{N_{b}} T^{\prime \prime}
\end{aligned}
$$

for an integer $m$

$$
\begin{gathered}
\chi_{m}=0 \text { for } m \leq 1 \\
=1 \text { for } m>1
\end{gathered}
$$

From $q=0$ to $q=1$, we can have

$$
\begin{array}{ll}
u(y ; 0)=u_{0}(y), & u(y ; 1)=u(y) \\
w(y ; 0)=w_{0}(y), & w(y ; 1)=w(y) \\
T(y ; 0)=T_{0}(y), & T(y ; 1)=T(y) \\
\phi(y ; 0)=\phi_{0}(y), & \phi(y ; 1)=\phi(y)
\end{array}
$$

Thus, as $q$ varies from 0 to $1, u(y, q) . w(y, q), T(y, q)$ and $\phi(y, q)$ varies continuously from $\mathrm{u}_{0}, \mathrm{w}_{0}, \mathrm{~T}_{0}$ and $\phi_{0}$ to the final solution $u(y) . w(y), T(y)$ and $\phi(y)$ respectively. Using Taylor's series and due to Eq. (21), $u . w, T$ and $\phi$ are be illustrated as

$$
\begin{gathered}
u(y ; q)=u_{0}(y)+\sum_{m=1}^{\infty} u_{m}(y) q^{m}, \quad u_{m}(\eta)=\left.\frac{1}{m !} \frac{\partial^{m} u(y ; q)}{\partial q^{m}}\right|_{q=0}, \\
w(y ; q)=w_{0}(y)+\sum_{m=1}^{\infty} w_{m}(y) q^{m}, \quad w_{m}(y)=\left.\frac{1}{m !} \frac{\partial^{m} w(\eta ; q)}{\partial q^{m}}\right|_{q=0}, \\
T(y ; q)=T_{0}(y)+\sum_{m=1}^{\infty} T_{m}(y) q^{m}, \quad T_{m}(\eta)=\left.\frac{1}{m !} \frac{\partial^{m} T(y ; q)}{\partial q^{m}}\right|_{q=0}, \\
\phi(y ; q)=\phi_{0}(y)+\sum_{m=1}^{\infty} \phi_{m}(y) q^{m}, \quad \phi_{m}(y)=\left.\frac{1}{m !} \frac{\partial^{m} \phi(y ; q)}{\partial q^{m}}\right|_{q=0} .
\end{gathered}
$$

We have to choose the values of the auxiliary parameters for which the series (25) are convergent at $q=1$ i.e.,

$$
\begin{aligned}
& u(y)=u_{0}(y)+\sum_{m=1}^{\infty} u_{m}(y), \quad w(y)=w_{0}(y)+\sum_{m=1}^{\infty} w_{m}(y), \\
& T(y)=T_{0}(y)+\sum_{m=1}^{\infty} T_{m}(y), \quad \phi(y)=\phi_{0}(y)+\sum_{m=1}^{\infty} \phi_{m}(y) .
\end{aligned}
$$




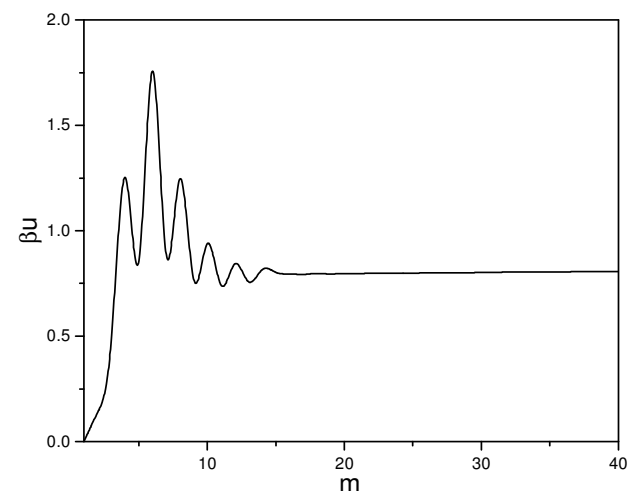

(a) $\beta_{U}$

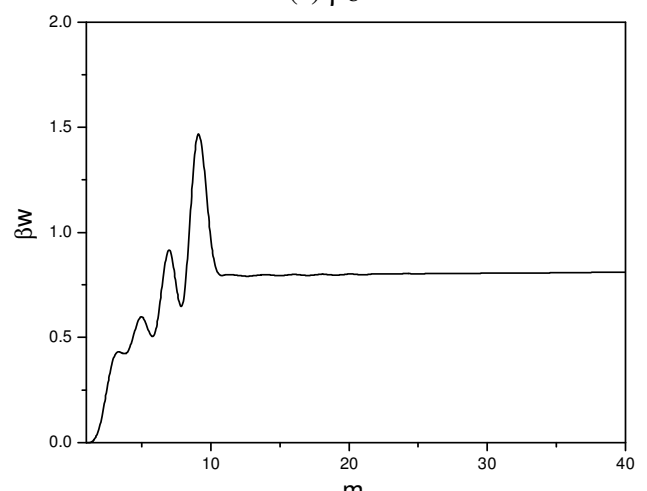

(b) $\beta_{\mathrm{w}}$

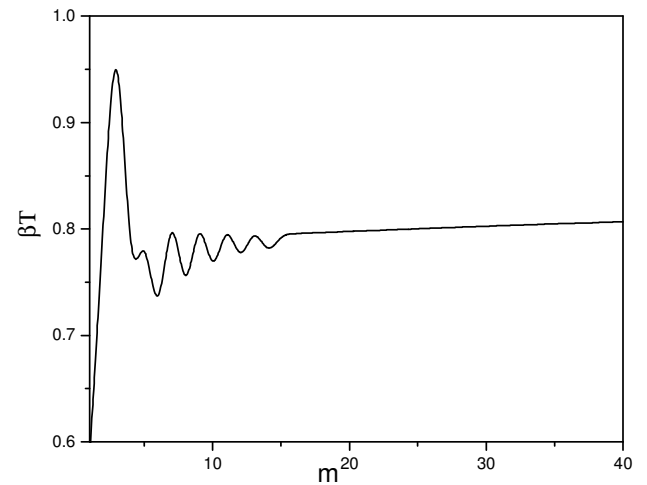

(c) $\beta_{\mathrm{T}}$

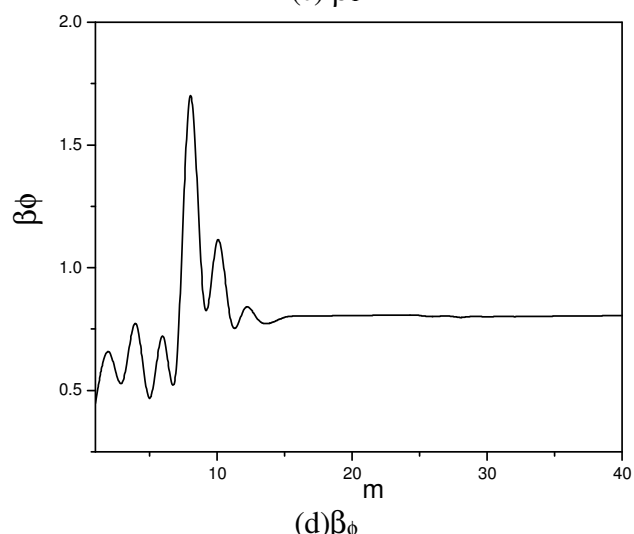

(d) $\beta_{\phi}$

Fig. 3 The ratio $\beta_{\mathrm{u}}, \beta_{\mathrm{w},} \beta_{\mathrm{T}}$ and $\beta_{\phi}$ to expose the convergence of the HAM solutions.

\section{CONVERGENCE}

In HAM, it is essential to see that the series solution converges. Also, the rate of convergence of approximation for the homotopy solution mainly depends on the values of $h$. To find the admissible space of the auxiliary parameters, h contours are drawn for a $20^{\text {th }}$ level of approximation and shown in Fig.1. It is evident from this figure that the admissible range for $\mathrm{h}_{1}, \mathrm{~h}_{2}, \mathrm{~h}_{3}$ and $\mathrm{h}_{4}$ are $-0.6<\mathrm{h}_{1}<-0.3,-0.65<\mathrm{h}_{2}<-0.4,-1.5<\mathrm{h}_{3}<-0.3$ and $-1.6<\mathrm{h}_{4}<-0.15$ respectively.

Table 1 Optimality of $h_{1}, h_{2}, h_{3}$ and $h_{4}$ at different order of approximations

\begin{tabular}{|c|ll|ll|}
\hline \multirow{2}{*}{ Order } & \multicolumn{2}{|c|}{ Optimal of $\mathrm{h}_{1}$} & \multicolumn{2}{l|}{ Optimal of $\mathrm{h}_{2}$} \\
\cline { 2 - 5 } & $\mathrm{h}_{1}$ & Min of $\mathrm{E}_{\mathrm{m}}$ & $\mathrm{h}_{2}$ & Min of $\mathrm{E}_{\mathrm{m}}$ \\
\hline 10 & -0.59 & $4.50 \times 10^{-6}$ & -0.51 & $8.43 \times 10^{-8}$ \\
\hline 15 & -0.59 & $8.81 \times 10^{-8}$ & -0.52 & $4.71 \times 10^{-10}$ \\
\hline 20 & -0.60 & $4.51 \times 10^{-8}$ & -0.51 & $8.52 \times 10^{-11}$ \\
\hline
\end{tabular}

\begin{tabular}{|c|ll|ll|}
\hline \multirow{2}{*}{ Order } & \multicolumn{2}{|c|}{ Optimal of $\mathrm{h}_{3}$} & \multicolumn{2}{c|}{ Optimal of $\mathrm{h}_{4}$} \\
\cline { 2 - 5 } & $\mathrm{h}_{3}$ & Min of $\mathrm{E}_{\mathrm{m}}$ & $\mathrm{h}_{4}$ & Min of $\mathrm{E}_{\mathrm{m}}$ \\
\hline 10 & -0.20 & $2.13 \times 10^{-8}$ & -1.56 & $6.26 \times 10^{-8}$ \\
\hline 15 & -0.20 & $4.80 \times 10^{-9}$ & -1.60 & $5.41 \times 10^{-11}$ \\
\hline 20 & -0.16 & $2.30 \times 10^{-9}$ & -1.60 & $8.69 \times 10^{-10}$ \\
\hline
\end{tabular}

The following average residual errors (Ref. Liao (2010) computed to obtain the optimal value of auxiliary parameter

$$
\begin{aligned}
& E_{u, m}=\frac{1}{2 K} \sum_{i=-K}^{K}\left(N_{1}\left[\sum_{j=0}^{m} u_{j}(i \Delta t)\right]\right)^{2}, \quad E_{w, m}=\frac{1}{2 K} \sum_{i=-K}^{K}\left(N_{2}\left[\sum_{j=0}^{m} w_{j}(i \Delta t)\right]\right)^{2}, \\
& E_{T, m}=\frac{1}{2 K} \sum_{i=-K}^{K}\left(N_{3}\left[\sum_{j=0}^{m} T_{j}(i \Delta t)\right]\right)^{2}, \quad E_{\phi, m}=\frac{1}{2 K} \sum_{i=-K}^{K}\left(N_{4}\left[\sum_{j=0}^{m} \phi_{j}(i \Delta t)\right]\right)^{2} .
\end{aligned}
$$

where $\Delta t=1 / K$, and $K=5$. At various levels of approximations (m), least average residual errors are represented in the Table. 1 . From this, we see that the average residual errors are least at $\mathrm{h}_{1}=-0.59, \mathrm{~h}_{2}=0.51, \mathrm{~h}_{3}=-$ 0.20 and $\mathrm{h}_{4}=-1.6$. Therefore, the optimality of convergence control parameters is appropriated as $\mathrm{h}_{1}=-0.59, \mathrm{~h}_{2}=0.51, \mathrm{~h}_{3}=-0.20$ and $\mathrm{h}_{4}=-$ 1.6.For different values of $\mathrm{m}$ the series solutions are calculated and represented in Table. 2. It is notice from this table that the series (25) converges in the total area of y. In the homotopy series the graphs of the ratio

$\beta_{u}=\left|\frac{u_{m}(h)}{u_{m-1}(h)}\right|, \quad \beta_{w}=\left|\frac{w_{m}(h)}{w_{m-1}(h)}\right|, \quad \beta_{T}=\left|\frac{T_{m}(h)}{T_{m-1}(h)}\right|, \quad \beta_{\phi}=\left|\frac{\phi_{m}(h)}{\phi_{m-1}(h)}\right|$. (28)

versus opposing the number of terms $m$ in the homotopy series is represented in Fig.3. These figures indicate that the series (26) converges to the exact solution.

Table 2 Convergence of HAM solutions for different order of approximations

\begin{tabular}{|c|c|c|c|c|}
\hline Order & $\mathrm{u}(0)$ & $\mathrm{w}(0)$ & $\mathrm{T}(0)$ & $\phi(0)$ \\
\hline 5 & 0.10620400 & 0.031283400 & 0.53412318 & 0.46716189 \\
\hline 10 & 0.10621315 & 0.030166000 & 0.54427627 & 0.46723565 \\
\hline 15 & 0.10621472 & 0.029013511 & 0.54731928 & 0.46734612 \\
\hline 20 & 0.10622485 & 0.029015717 & 0.54731934 & 0.46735634 \\
\hline 25 & 0.10622487 & 0.029016788 & 0.54731936 & 0.46735657 \\
\hline 30 & 0.10622495 & 0.029023823 & 0.54732937 & 0.46735667 \\
\hline 35 & 0.10622496 & 0.029025837 & 0.54732938 & 0.46736671 \\
\hline 40 & 0.10622497 & 0.029025838 & 0.54732938 & 0.46736774 \\
\hline 45 & 0.10622498 & 0.029026893 & 0.54732939 & 0.46736775 \\
\hline 50 & 0.10622498 & 0.029026893 & 0.54732939 & 0.46736879 \\
\hline 55 & 0.10622498 & 0.029026893 & 0.54732939 & 0.46736879 \\
\hline
\end{tabular}




\section{RESULTS AND DISCUSSION}

The influence of magnetic parameter, Hall, ion-slip parameters, Buoyancy ratio parameter and thermophoresis parameter on $\mathrm{u}(\mathrm{y}), \mathrm{w}(\mathrm{y})$, $\mathrm{T}(\mathrm{y})$ and $\phi(y)$ are represented graphically in Figures 4-7. To study these effect of these parameters, the computations were carried out by taking $\mathrm{Le}=1.0, \mathrm{Br}=0.5, \mathrm{Pr}=1.0, \mathrm{Gr}=10, \mathrm{R}=1, \mathrm{Re}=2, \mathrm{Nb}=0.5$, and $\mathrm{A}=$ 1 .

Figure 4 exhibits the impact of the magnetic parameter $\mathrm{Ha}$ on velocity in flow direction, induced flow velocity, temperature and nanoparticle concentration. Figure (4a) reveals that the dimensionless velocity is decreasing with the increase in Ha. The transverse magnetite field orthogonal to the flow direction gives a resistance force called as Lorentz force. This Lorentz force resists the fluid flow and due to this velocity in flow direction decrease. The influence of $\mathrm{Ha}$ on is represented in figure 4(b). It is depicted from this figure that is increasing with a rise in Ha. Figure 4(c) explains the changes of dimensionless temperature with Ha. The temperature is decaying with the growth in the parameter Ha. Figure 4(d) interprets the variations of nanoparticle concentration with Ha. The nanoparticle concentration is reduced with an enhancement in the magnetite parameter. The resistance, created by the Lorentz force, increases the friction between its fluid layers hence decrease the temperature and nanoparticle concentration.

The changes of velocity in flow direction, induced flow velocity, temperature and nanoparticle concentration with $\beta_{h}$ is presented in Figure 5. It is identified from Figure 5(a) that the velocity is increasing with the rise in $\beta_{h}$. Figure 5(b) reveals that the induced flow in the direction of the $\mathrm{z}$-axis is decreasing as the value of the Hall parameter raises. From Figure 5(c), it is noticed that the temperature is diminishing with the rise in $\beta_{h}$. Nanoparticle concentration is decreasing with an increase in $\beta_{h}$ as depicted in Figure 5(d). The inclusion of Hall currents reduces the effective conductivity and hence drops the resisting force enforced by the magnetic field. Hence the increase in the Hall parameter enhances the velocity component and temperature.

The variation of dimensionless velocity, induced flow velocity, temperature and nanoparticle concentration with $\beta_{i}$ is shown in Figure 6. It is seen from the Figure 6(a) that the velocity in flow direction is increasing with an enhancement in the parameter $\beta_{i}$. Figure 6(b) reveals that the induced velocity flow is decaying as the ion-slip parameter enhances. From Figure 6(c), it is noticed that the temperature is diminishing with the rise in $\beta_{i}$. There is a decay in the nanoparticle concentration with the rise in $\beta_{i}$ as depicted in Figure 6(d).

Figure 7 represents the influence of $\mathrm{Nr}$ on velocity in flow direction, induced flow velocity, temperature and nanoparticle concentration. From Figure 7(a), it is identified that the velocity is increasing with the rise in Nr. Figure 7(b) explains that the induced flow in the direction of z-axes is diminishing with the rise in the parameter $\mathrm{Nr}$. The dimensionless temperature is rising with the rise in the parameter $\mathrm{Nr}$ as shown in Figure 7(c). From figure (7d), it is noticed that the nanoparticle concentration is decreasing with the enhancement in the parameter $\mathrm{Nr}$.

The significance of $\mathrm{Nt}$ on velocity in flow direction, induced flow velocity, temperature and nanoparticle concentration is represented in Figure 8. The velocity is increasing with the rise in $\mathrm{Nt}$ as shown in Figure 8(a). An increase of Nt lead to increase in the effective conductivity, in turn, decrease in the damping force. It is observed from Figure 8(b) that the induced flow in the direction of the z-axes is diminishing with the enhancement in Nt. Figure 8(c) reveals that the temperature is increasing with the enhance in Nt. From Figure 8(d), it is noticed that the nanoparticle concentration is decaying with the enhancement in $\mathrm{Nt}$.

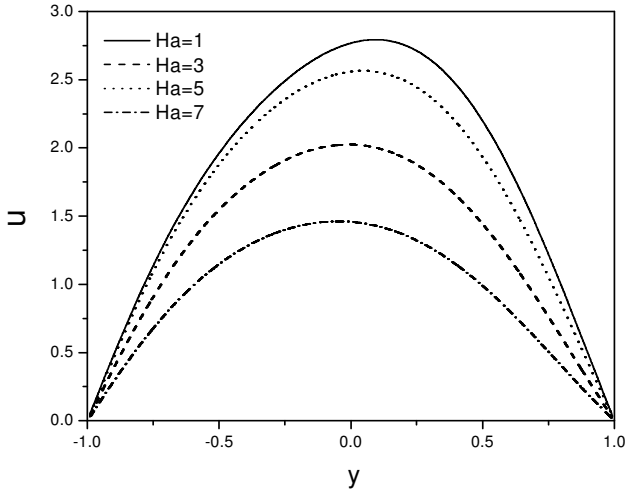

(a) $\mathrm{u}(\mathrm{y})$

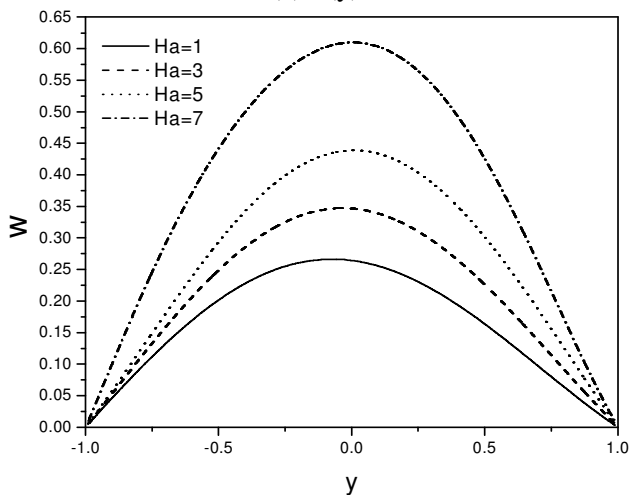

(b) w(y)

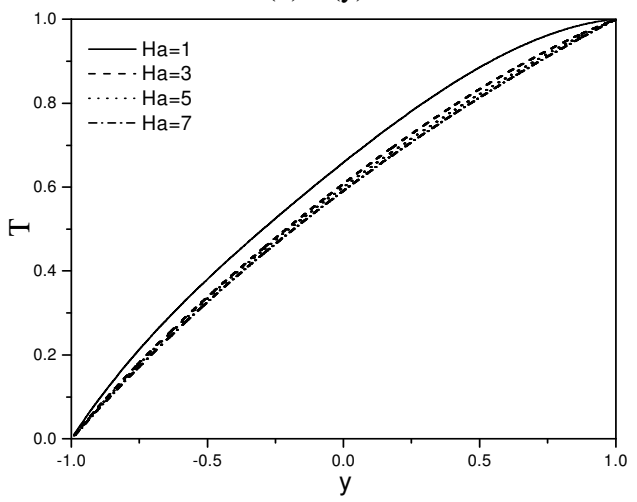

(c) $\mathrm{T}(\mathrm{y})$

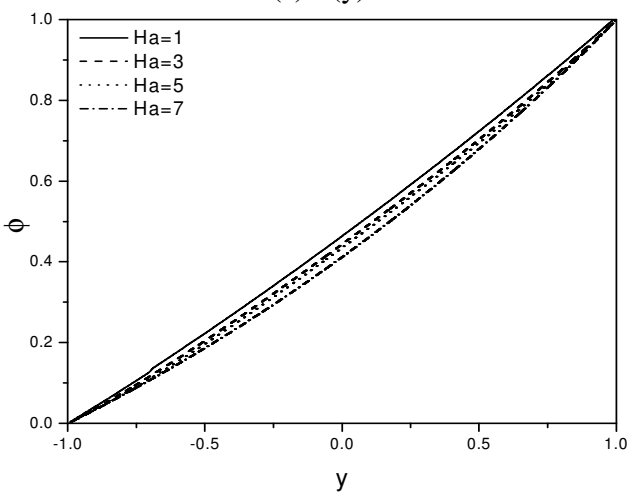

(d) $\phi(y)$

Fig. 4 Effect of $H a$ on $\mathrm{u}(\mathrm{y}), \mathrm{w}(\mathrm{y}), \mathrm{T}(\mathrm{y})$ and $\phi(\mathrm{y})$ for $\mathrm{Le}=1.0, \mathrm{Br}=0.5, \mathrm{Pr}$ $=1.0, \mathrm{Gr}=10, \mathrm{R}=1, \mathrm{Re}=2, \mathrm{Nt}=0.5, \mathrm{Nb}=0.5, \mathrm{Sr}=0.5, \mathrm{Nr}=1.0, \beta_{\mathrm{i}}=$ $2.0, \beta_{\mathrm{h}}=2.0$ and $\mathrm{A}=1$. 


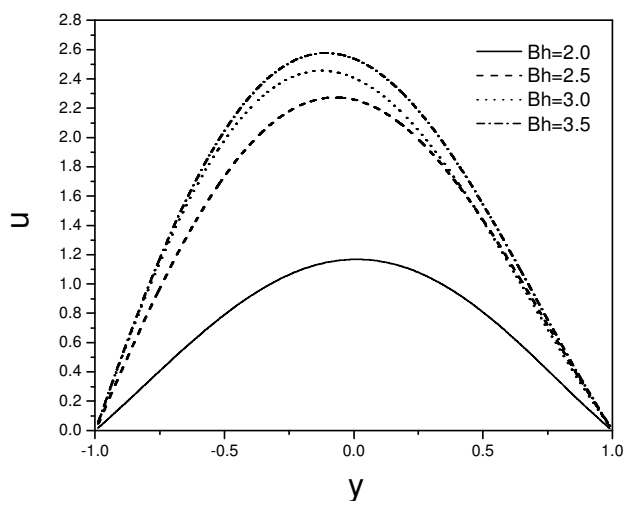

(a) $u(y)$

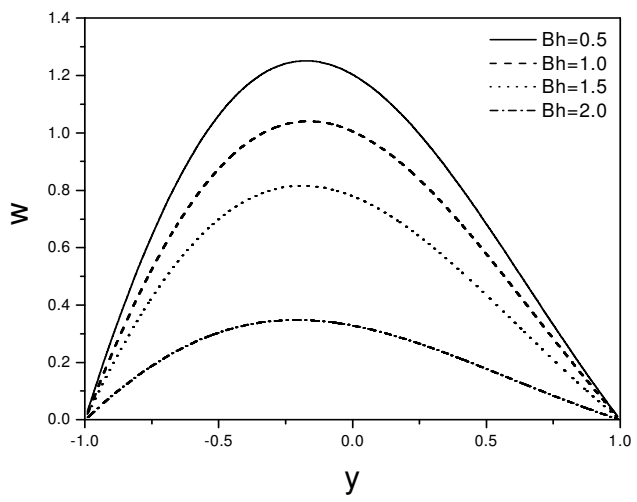

(b) $w(y)$

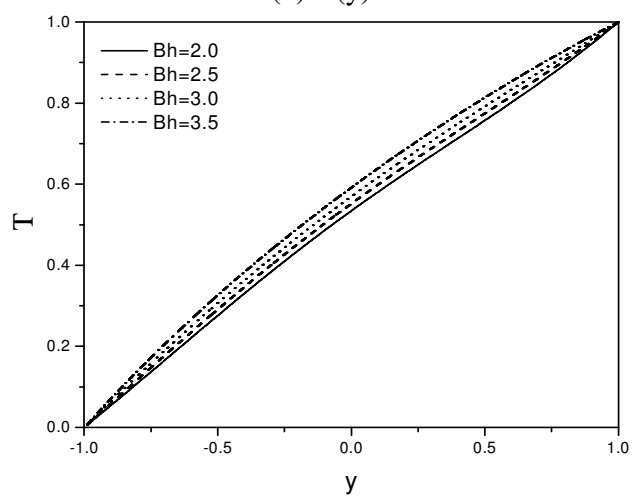

(c) $\mathrm{T}(\mathrm{y})$

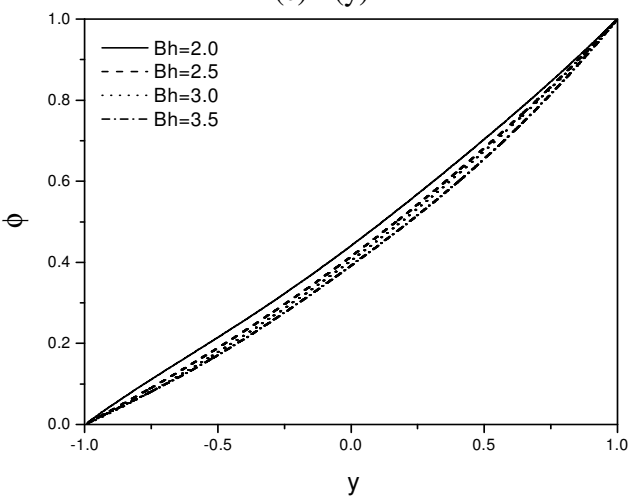

(d) $\phi(y)$

Fig. 5 Effect of $\beta_{\mathrm{h}}$ on $\mathrm{u}(\mathrm{y}), \mathrm{w}(\mathrm{y}), \mathrm{T}(\mathrm{y})$ and $\phi(\mathrm{y})$ for $\mathrm{Le}=1.0, \mathrm{Br}=0.5$, $\mathrm{Pr}=1.0, \mathrm{Gr}=10, \mathrm{R}=1, \mathrm{Re}=2, \mathrm{Nr}=1.0, \mathrm{Nt}=0.5, \mathrm{Nb}=0.5, \mathrm{Sr}=0.5$, $\mathrm{Ha}=4.0, \beta_{\mathrm{i}}=2.0$ and $\mathrm{A}=1$.

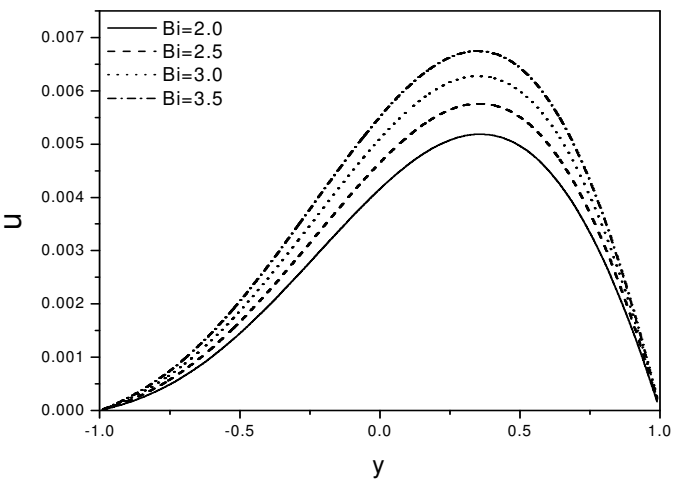

(a) $\mathrm{u}(\mathrm{y})$

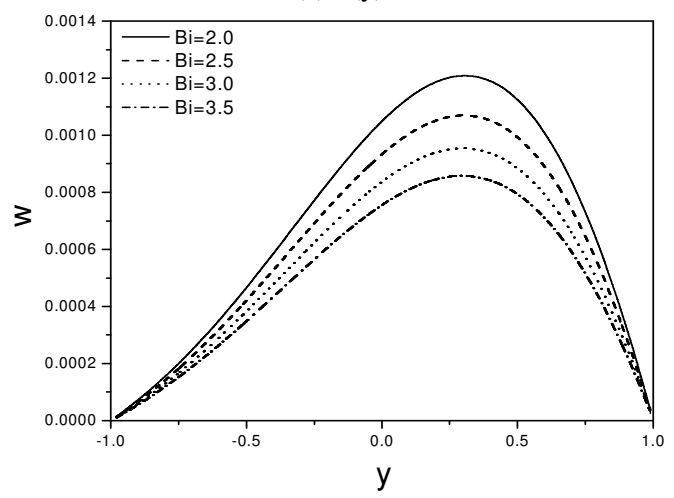

(b) w(y)

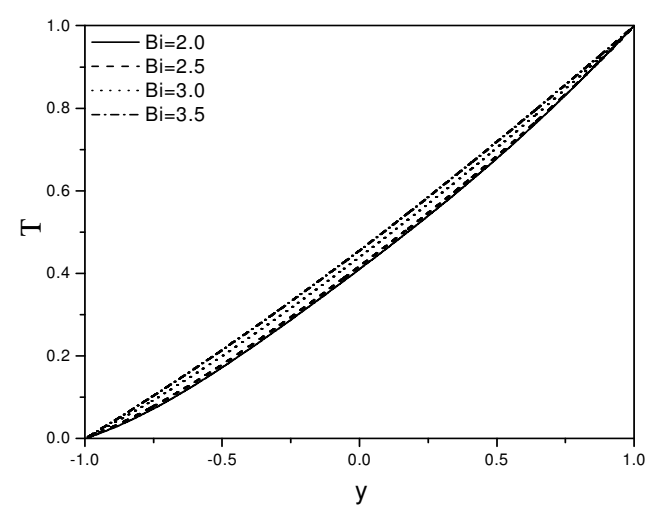

(c) $\mathrm{T}(\mathrm{y})$

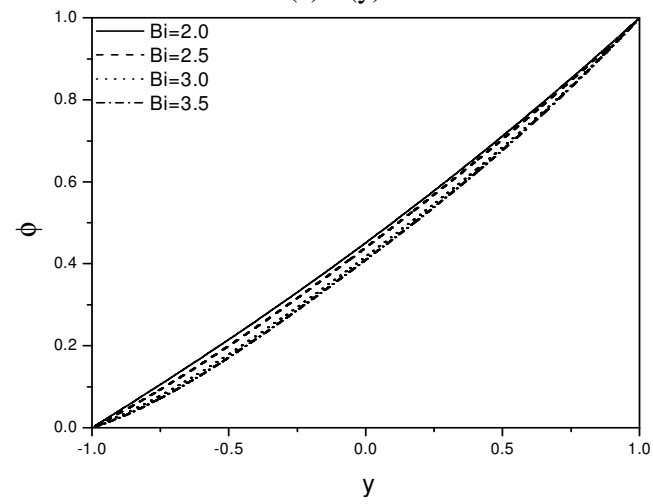

(d) $\phi(y)$

Fig. 6 Effect of $\beta_{\mathrm{i}}$ on $\mathrm{u}(\mathrm{y}), \mathrm{w}(\mathrm{y}), \mathrm{T}(\mathrm{y})$ and $\phi(\mathrm{y})$ for $\mathrm{Le}=1.0, \mathrm{Br}=0.5, \mathrm{Pr}$ $=1.0, \mathrm{Gr}=10, \mathrm{R}=1, \mathrm{Re}=2, \mathrm{Nr}=1.0, \mathrm{Nt}=0.5, \mathrm{Nb}=0.5, \mathrm{Sr}=0.5, \mathrm{Ha}=$ $4.0, \beta_{\mathrm{h}}=2.0$ and $\mathrm{A}=1$. 


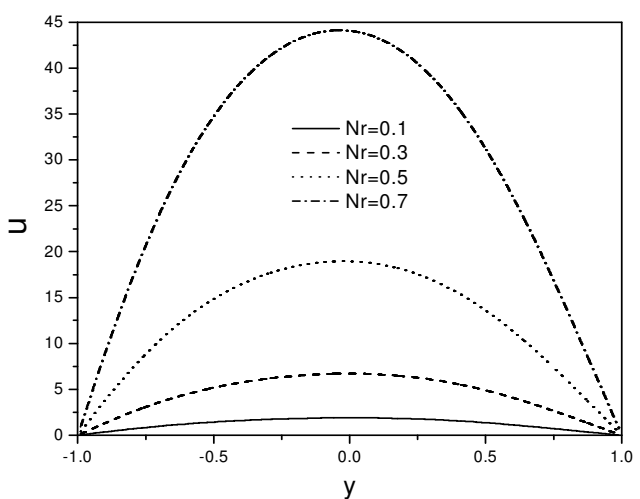

(a) $u(y)$

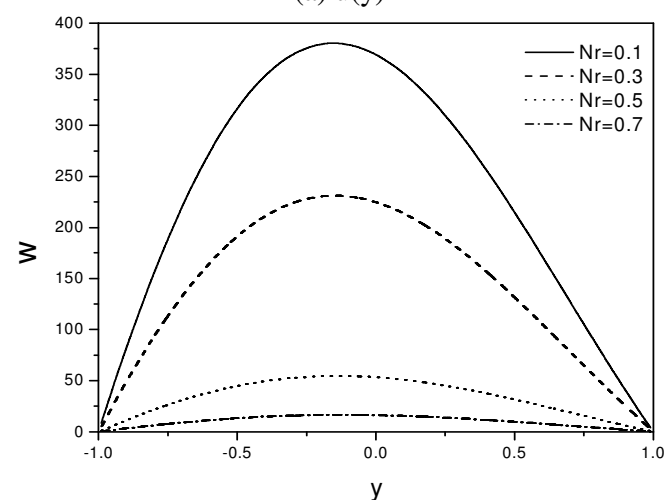

(b) w(y)

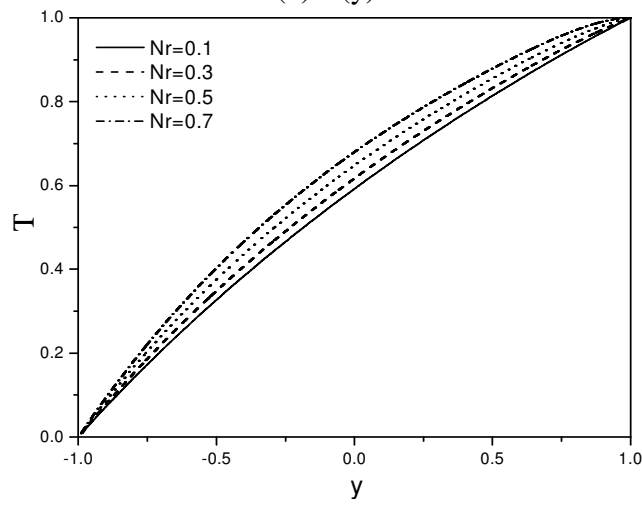

(c) $\mathrm{T}(\mathrm{y})$

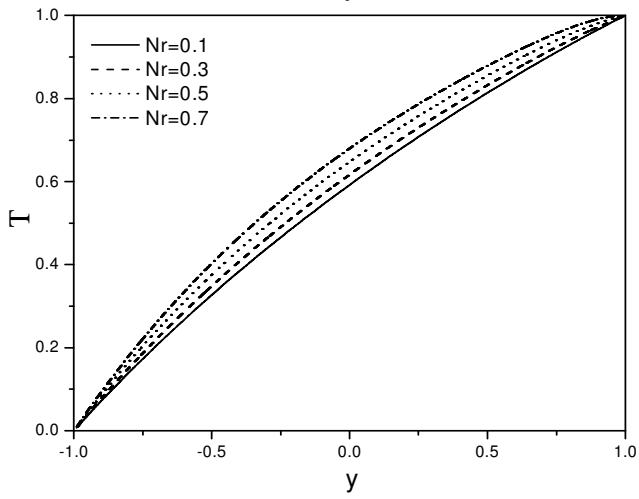

(d) $\phi(y)$

Fig. 7 Effect of $\mathrm{Nr}$ on $\mathrm{u}(\mathrm{y}), \mathrm{w}(\mathrm{y}), \mathrm{T}(\mathrm{y})$ and $\phi(\mathrm{y})$ for $\mathrm{Le}=1.0, \mathrm{Br}=0.5$, $\mathrm{Pr}=1.0, \mathrm{Gr}=10, \mathrm{R}=1, \mathrm{Re}=2, \mathrm{Nt}=0.5, \mathrm{Nb}=0.5, \mathrm{Sr}=0.5, \mathrm{Ha}=4.0, \beta_{\mathrm{i}}$ $=2.0, \beta_{\mathrm{h}}=2.0$ and $\mathrm{A}=1$.

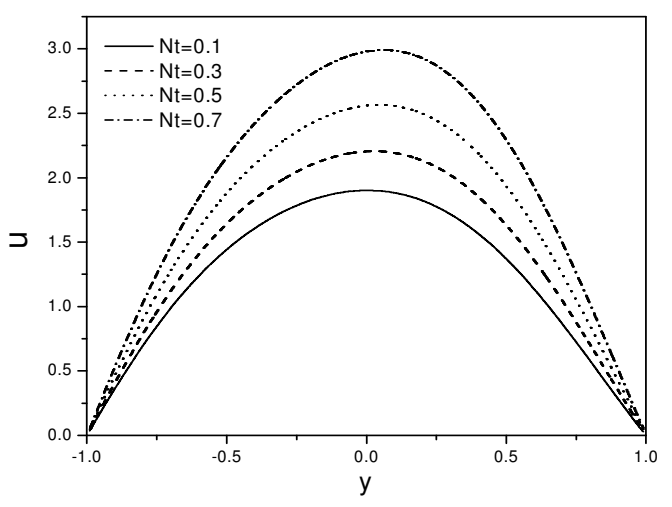

(a) $\mathrm{u}(\mathrm{y})$

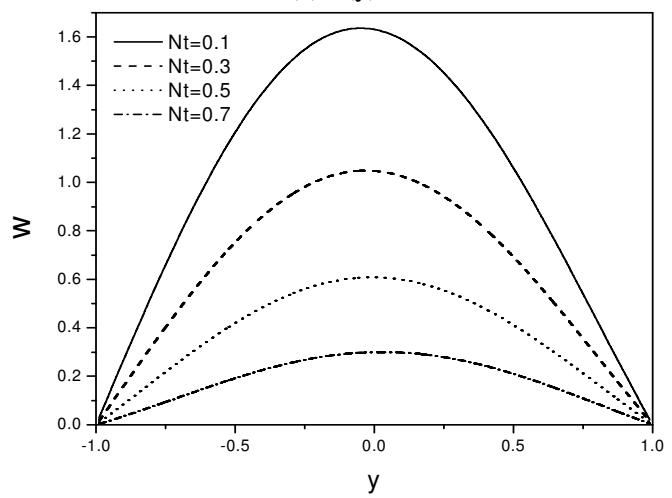

(b) $\mathrm{w}(\mathrm{y})$

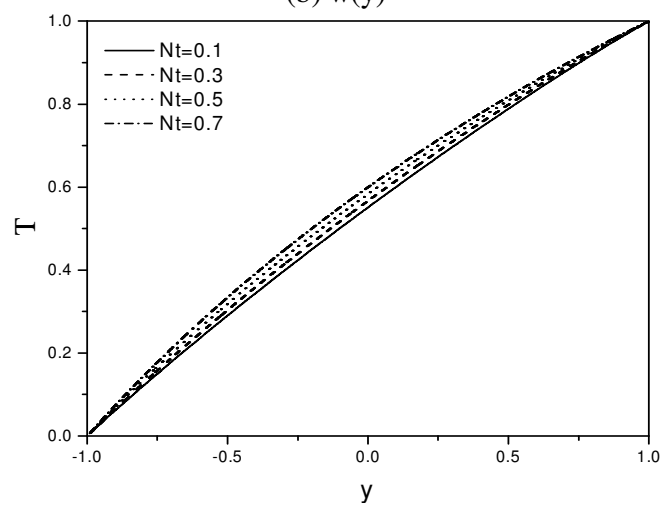

(c) $\mathrm{T}(\mathrm{y})$

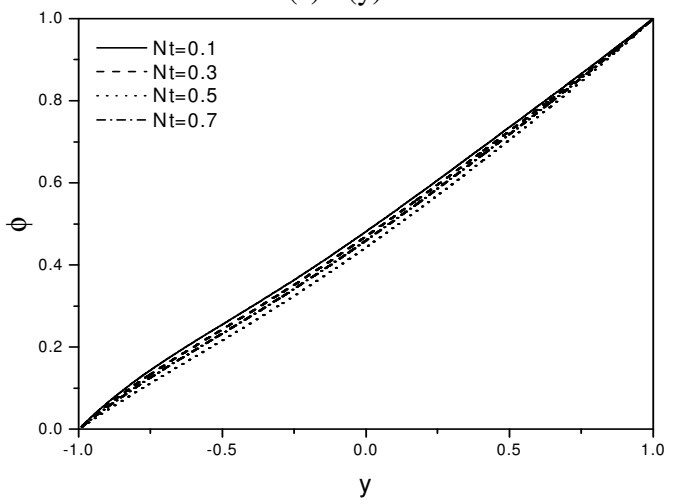

(d) $\phi(y)$

Fig. 8 Effect of Nton $\mathrm{u}(\mathrm{y}), \mathrm{w}(\mathrm{y}), \mathrm{T}(\mathrm{y})$ and $\phi(\mathrm{y})$ for $\mathrm{Le}=1.0$, $\mathrm{Br}=0.5, \mathrm{Pr}=1.0, \mathrm{Gr}=10, \mathrm{R}=1, \mathrm{Re}=2, \mathrm{Nr}=1.0, \mathrm{Nb}=0.5$, $\mathrm{Sr}=0.5, \mathrm{Ha}=4.0, \beta_{\mathrm{i}}=2.0, \beta_{\mathrm{h}}=2.0$ and $\mathrm{A}=1$. 


\section{CONCLUSIONS}

The present study investigates the influence of Hall current, Ion slip, thermoporesis and buoyancy ratio on mixed convection flow of nanofluid in a vertical channel. Using HAM the non-dimensional nonlinear equations are solved. The main decisions are encapsulated below:

- The velocity, temperature, nanoparticle concentration decreases whereas the velocity in $\mathrm{z}$-direction raises with the increment in magnetic parameter.

- As Hall parameter increases, the dimensionless velocity and temperature rises, but the induced flow velocity and nanoparticle concentration decreased.

- As Ion-slip parameter raises, the velocity and temperature increases, but the induced flow velocity and nanoparticle concentration decreases.

\section{NOMENCLATURE}

\begin{tabular}{ll}
$\mathrm{A}$ & Constant pressure gradient \\
$\mathrm{Br}$ & Brinkman number \\
$\mathrm{C}_{\mathrm{p}}$ & Specific heat capacity \\
$\mathrm{D}$ & Mass diffusivity \\
$\mathrm{D}$ B & Brownian diffusion coefficient \\
$\mathrm{D}$ T & Thermophoretic diffusion coefficient \\
$\mathrm{Gr}$ & Grashof number \\
$\mathrm{Ha}$ & Hartman number \\
$\mathrm{Le}$ & Lewis number \\
$\mathrm{Nb}$ & Brownian motion parameter \\
$\mathrm{Nr}$ & Buoyancy ratio is \\
$\mathrm{Nt}$ & Thermoporesis parameter \\
$\mathrm{Pr}$ & Prandtl number \\
$\mathrm{R}$ & suction/injection parameter \\
$\mathrm{Re}$ & Reynold's number \\
$\beta_{h}$ & Hall parameter \\
$\beta_{i}$ & Ion-slip parameter \\
$\beta_{T}$ & Coefficients of thermal expansion \\
$\rho$ & Density of the fluid \\
$g$ & Acceleration due to gravity \\
$\mu$ & Viscosity coefficient \\
$\sigma$ & Electrical conductivity \\
$\alpha$ & Effective thermal diffusivity \\
$\mathrm{k}$ & thermal conductivity \\
$v$ & Kinematic viscosity coefficient \\
$\mathrm{u}$ & Velocity \\
$\mathrm{v}$ & Induced flow velocity \\
$\mathrm{T}$ & Temperature \\
$\phi$ & Nanoparticle concentration \\
& \\
\hline
\end{tabular}

\section{REFERENCES}

Buongiorno, J., 2006, “Convective Transport in Nanofluids,"ASME J. Heat Transfer, 128, 240 - 250. http://dx.doi.org/10.1115/1.2150834

Choi, S.U.S., 1995, "Enhancing Thermal Conductivity of Fluids with Nanoparticles," In Singer, D and Wang, H (Eds.), Development and Applications of Non-Newtonian Flows, American Society of Mechanical Engineers, New York, 99-106.

Das, S. and Jana, R.N., 2015, "Natural Convective Magneto Nanofluid Flow and Radiative Heat Transfer Past A Moving Vertical Plate," Alexandria Eng. J., 54(1), 55-64.

http://dx.doi.org/10.1016/j.aej.2015.01.001

Fakour, M., Vahabzadeh, A. and Ganji, D.D., 2104, "Scrutiny of Mixed Convection Flow of a Nanofluid in a Vertical Channel," Case Stud. Therm. Eng., 4, 15-23.

http://dx.doi.org/10.1016/j.csite.2014.05.003
Garg, B.P., Singh, K.D. and Bansal A.K., 2014, "Hall Current Effect on Viscoelastic (Walters Liquid Model-B) MHD Oscillatory Convective Channel Flow through a Porous Medium with Heat Radiation," Kragujevac Journal of Science, 36, 19-32.

Grosan, T., Pop, I., 2012, "Fully Developed Mixed Convection in a Vertical Channel Filled by a Nanofluid," J. Heat Transfer, 134(8), 082501 .

http://dx.doi.org/10.1115/1.4006159

Hajipour, M., Dehkordi, A.M., 2012, “Analysis of Nanofluid Heat Transfer in Parallel Plate Vertical Channels Partially Filled with Porous Medium," Int. J. Thermal Sciences, 55, 103-113.

http://dx.doi.org/10.1016/j.ijthermalsci.2011.12.018

Hang, X., Tao, F. and Pop, I., 2013, “Analysis of Mixed Convection Flow of a Nanofluid in a Vertical Channel with the Buongiorno Mathematical Model", Int. Commu. Heat Mass Transfer, 44, pp. 15-22.

http://dx.doi.org/10.1016/j.icheatmasstransfer.2013.03.015

Hang, X., Pop, I., 2012, "Fully Developed Mixed Convection Flow in a Vertical Channel Filled with Nanofluids," Int. Commun. Heat Mass

Transfer, 39, 1086-1092

http://dx.doi.org/10.1016/j.icheatmasstransfer.2012.06.003

Liao, S.J., 2010, “An Optimal Homotopy Analysis Approach for Strongly Nonlinear Differential Equations," Communications in Nonlinear Science and Numerical Simulation, 15(8), 2003-2016. http://dx.doi.org/10.1016/j.cnsns.2009.09.002

Liao S.J., 2003, Beyond Perturbation - Introduction to Homotopy Analysis Method. Boca Raton: Chapman and Hall/CRC Press.

Liao, S.J., 2004, "On the homotopy Analysis Method for Nonlinear Problems," Appl Math Comput 147(2), 499--513. http://dx.doi.org/10.1155/2011/237045

Liao, S.J. 2013, Advances in the Homotopy Analysis Method, World Scientific Publishing Company, Singapore.

Makhatar N.A.M, Saleh, H. and Ishak, H., 2015, "Flow Reversal of Fully Developed Mixed Convection of Nanofluids in a Vertical Channel Filled with Porous Medium," International Journal of Pure and Applied Mathematics, 103(1), 81-97. http://dx.doi.org/10.12732/ijpam.v103i1.7

Manglesh A. and Gorla M.G., 2013, "MHD Free Convection Flow through Porous Medium in the Presence of Hall Current, Radiation and Thermal Diffusion," Indian J. Pure Appl. Math., 44(6), 743-756. http://dx.doi.org/10.1007/s13226-013-0040-9

Sacheti, N.C., Chandran, P., Singh A. K. and Bhadauria, B.S., 2012, "Transient Free Convective Flow of a Nanofluid in a Vertical Channel," Int. J. Energy Technol. 4, 1-7.

Sheikholeslami, M. andGanji, D. D., 2014, "Magnetohydrodynamic Flow in a Permeable Channel Filled with Nanofluid," Sci. Iran., B21 (1), 203-212.

Srinivasacharya, D. and Kaladhar, K., 2012, "Mixed Convection Flow of Couple Stress Fluid between Parallel Vertical Plates with Hall and Ionslip Effects," Commun Nonlinear Sci Numer Simulat, 17, 2447-2462. http://dx.doi.org/10.1016/j.cnsns.2011.10.006

Srinivasacharya, D. and Kaladhar, K., 2013, “Analytical Solution for Hall and Ion-slip Effects on Mixed Convection Flow of Couple Stress Fluid between Parallel Disks," Mathematical and Computer Modelling, 57, 2494-2509.

http://dx.doi.org/10.1016/j.mcm.2012.12.036

Tani, I., 1962, "Steady Flow Of Conducting Fluids in Channels under Transverse Magnetic Fields with Consideration of Hall Effects," Journal of Aerospace Science, 29, 297-305.

http://dx.doi.org/10.2514/8.9412 